\title{
Reply to "Comment on 'Penetration of Action Potentials During Collision in the Median and Lateral Giant Axons of Invertebrates",
}

\author{
Tian Wang, Alfredo Gonzalez-Perez, Rima Budvytyte, Andrew D. Jackson, and Thomas Heimburg* \\ The Niels Bohr Institute, University of Copenhagen, Blegdamsvej 17, 2100 Copenhagen, Denmark
}

(Received 13 December 2016; published 24 April 2017)

\begin{abstract}
Berg et al. did not reproduce our results but worked on different preparations and, in one central experiment, used a significantly different electrode configuration. To clarify the situation, we have repeated their experiment on the walking leg of a lobster using an apparatus that can produce both electrode configurations. With the configuration used by Berg et al., the signal of the nerve pulse disappears when forced to pass through the region strongly perturbed by the second stimulus. In our original collision setup, pulses do not travel through perturbed regions, and pulses pass through each other without annihilation as previously reported. These results demonstrate that we handle the preparations correctly. Furthermore, they call for a reinterpretation of the so-called collision block experiment performed by Berg et al. Most likely, their results merely indicate inhibition of the nerve pulse by a strong stimulus and not annihilation upon collision as claimed.
\end{abstract}

DOI: 10.1103/PhysRevX.7.028002

In our article from 2014 [1] (referred to as GP2014 henceforth), we showed that pulses in earthworm axons and in the abdominal ventral cord of a lobster can pass through each other. We have since reproduced these results in lobster connectives when individual axons were cut [2]. No ganglia were present in the latter preparation. To our knowledge, the only previous work investigating this effect was that of Tasaki [3] who reported penetration of action potentials in motor nerves from toads. These results are important since predictions using the soliton theory for nerve pulses [4] differ from those of the Hodgkin-Huxley model [5]. The Comment by Berg et al. [6] (referred to as Berg2017 henceforth) questions both our experiments and their interpretation. The statement in our original paper (GP2014) was as follows: "We can falsify the general belief that annihilation must always occur because of the presence of a refractory period." We did not state that action potentials always pass through each other. In their comment, Berg2017 used either different preparations or different electrode configurations. Since neither case represents a repetition of our experiments, there is no basis for them to challenge our conclusion. For instance, they used living earthworms rather than isolated axons. Furthermore, they used the walking leg of a lobster rather than ventral cords, and they used a different electrode arrangement that leads to a different experimental result. Consequently, our

\footnotetext{
*theimbu@nbi.ku.dk
}

Published by the American Physical Society under the terms of the Creative Commons Attribution 4.0 International license. Further distribution of this work must maintain attribution to the author(s) and the published article's title, journal citation, and DOI.
Subject Areas: Biological Physics, Medical Physics conclusion has not been shown to be incorrect. We show here that pulses may pass through each other even in their setup. Our focus in GP2014 was on the difference between dissipative Hodgkin-Huxley action potentials and nondissipative solitary waves. We reply only to statements by Berg2017 that are related to our paper.

We respond to the points by Berg2017 in the order of appearance in their comment. Additionally, we provide several new experiments that support our original point.

(1) Berg2017 report that colliding pulses traveling in opposite directions in their experimental configuration annihilate in intact earthworms. This does not contradict GP2014, which studied extracted nerves and not intact animals. The experiment by Berg2017 may well be correct, but it does not provide sufficient grounds to question the validity of ours.

(2) The authors state that the action potential in earthworms is of an all-or-none nature. This assertion may be true but was neither challenged nor even addressed in our article. Furthermore, Berg2017 claim that the pulse amplitudes must be the same in both directions. However, in contrast to their assumption, they do not measure amplitudes. Both Berg2017 and GP2014 measure the difference in voltage between two closely spaced electrodes, which effectively corresponds to the spatial derivative of the amplitude. This derivative is zero at the maximum of the voltage pulse. The amplitude itself (i.e., the integral of the signal) is expected to be constant only for the HH model and only for the case of isotropic channel densities. In experiments with isolated nerves, the signal also depends on the quality of the contact between nerves and electrodes 
and is subject to additional variations. This has no effect on the propagation phenomenon itself. The criticism of Berg2017 is not justified.

(3) The authors state that velocities must be the same in both directions. This point is not relevant for the message of our 2014 paper. Determination of the velocities in that paper was intended to show that the velocity of individual pulses and of pulses after collision are the same within experimental error, which is true independent of any technical concerns raised by Berg2107. The statement by Berg2017 is also incorrect. In both the HH model and the soliton theory, pulse velocity depends, among other factors, on the diameter of the axon, which changes over the length of the earthworm or neurons. It is also incorrect that the reported pulse velocity in earthworm axons is "agreed" to be $20 \mathrm{~m} / \mathrm{s}$. In fact, it ranges from $3 \mathrm{~m} / \mathrm{s}$ to $30 \mathrm{~m} / \mathrm{s}$ depending on the nature and extension of the axon. Our data are in full agreement with the relevant literature on earthworm axons as described in Ref. [7].

(4) Berg2017 argue that compound action potentials in the walking leg of a lobster (and in the sciatic nerve of a frog) annihilate. Unfortunately, their electrode configuration differs significantly from ours. We show below that this leads to a different outcome of the experiment and most likely to a misinterpretation of their result. Since the authors did not reproduce our experiment, we decided to reproduce theirs and confirm ours at the same time. The electrode configuration of the experiment performed by Berg2017 on the nerve bundles from walking legs is shown in Fig. 1(b). Signals arising from the stimulation by electrodes $S_{1}$ and $S_{2}$ are recorded at one end by the recording electrodes $R$. Thus, in this configuration, only orthodromic pulses are measured. The assumption is that two pulses moving in opposite directions are generated at $S_{2}$. In order to arrive at the recording site $R$, the signal from $S_{1}$ must pass both the region of pulse collision and the perturbed region at stimulation site $S_{2}$. The collision of pulses and their possible annihilation are not measured directly but inferred from the absence of the signal from $S_{1}$ in the recording. Using the nomenclature of Berg2017, we call this experiment the "collision block experiment." In contrast, our electrode configuration measures the collision directly. In the following, we call this the "collision experiment." The recorded pulses do not pass through a stimulated or a perturbed region [Fig. 1(a)]. The simple interchange of $R$ and $S_{2}$ is sufficient to switch from one electrode configuration to the other. Berg2017 did not perform or report this simple control experiment. Panel (a) shows the recordings using the configuration from GP2014; panel (b) shows the recording on the same nerve following the (a)

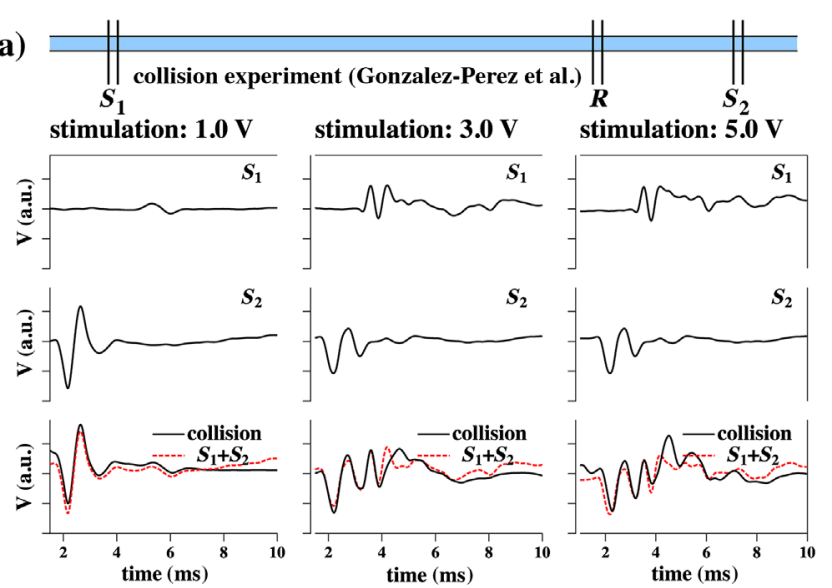

(b)


FIG. 1. (a) The recording electrodes are between stimulation sites $S_{1}$ and $S_{2}$. We show the signals from $S_{1}$ only (top row), $S_{2}$ only (middle row), and the collision experiment (bottom row) at three different stimulation voltages. At all voltages, the sum of the individual signals from $S_{1}$ and $S_{2}$ and the signal after simultaneous stimulation are very similar or identical. This result implies that the pulses have passed through each other. (b) The experiment by Berg2017 (recording site at the distal end of the nerve after stimulation sites $S_{1}$ and $S_{2}$ ) shows a different outcome. While the sum of the individual signals is identical to the postcollision pulse for a stimulation of $1 \mathrm{~V}$, the signal from $S_{1}$ is increasingly inhibited for higher voltages.

switching of the electrodes to the configuration of Berg2017. It is apparent that the signal originating from $S_{1}$ disappears at high stimulation voltages in the configuration used by Berg2017, while pulses both from $S_{1}$ and $S_{2}$ remain in the recordings using the configuration of GP2014. At low voltage and without exception, signals from both $S_{1}$ and $S_{2}$ could be seen at the recording site in Berg's electrode configuration in all experiments on many nerves. Since we can reproduce the results of Berg2017 while simultaneously confirming our own results, we have shown that (i) we treat our nerves correctly and (ii) the collision experiment and the collision block experiment do not measure the same phenomenon. We believe that the 
strongly perturbed region at stimulation site $S_{2}$ of the collision block experiment [panel (b)] does not permit propagation of a pulse from $S_{1}$ even if orthodromic and antidromic pulses do pass through each other prior to the arrival of the signal from $S_{1}$ at $S_{2}$. In Ref. [7], we repeat this experiment using two pairs of recording electrodes so that both experiments are performed simultaneously (Fig. S1). This experiment confirms the findings shown in Fig. 1.

(5) As a consequence of point 4, the final statement by Berg2017 ("annihilation has been reported in dozens, probably hundreds, of publications over the course of more than 65 years") is possibly not correct. As shown here, the so-called collision block experiment probably measures something else. This does not mean that these experiments are not correct-it just means that one may have to interpret them differently. The respective literature should therefore be revisited. As shown here, it is possible that the collision block experiment (described in GP2014 and Berg2017) rather indicates the inhibition of nerves at the site of a very strong stimulus without addressing the question of annihilation.

Conclusion.-For the lobster walking leg, we have shown that both the collision block experiment of Berg2017 [6] and our collision experiment can be reproduced in the same nerve. Since collision cannot simultaneously lead to annihilation and to penetration, our results suggest that Berg2017 have measured inhibition of the nerve at the stimulation site and not annihilation. Since we reproduce their result, we further demonstrate that we handle the nerve correctly.
The experiments performed by Berg2017 [6] were not identical repeats of our original experiments. Therefore, their results do not invalidate ours.

This work was supported by the Villum Foundation (VKR 022130).

[1] A. Gonzalez-Perez, R. Budvytyte, L. D. Mosgaard, S. Nissen, and T. Heimburg, Penetration of Action Potentials During Collision in the Median and Lateral Giant Axons of Invertebrates, Phys. Rev. X 4, 031047 (2014).

[2] A. Gonzalez-Perez, L. D. Mosgaard, R. Budvytyte, E. Villagran Vargas, A. D. Jackson, and T. Heimburg, Solitary Electromechanical Pulses in Lobster Neurons, Biophys. Chem. 216, 51 (2016).

[3] I. Tasaki, Collision of Two Nerve Impulses in the Nerve Fiber, Biochim. Biophys. Acta 3, 494 (1949).

[4] T. Heimburg and A. D. Jackson, On Soliton Propagation in Biomembranes and Nerves, Proc. Natl. Acad. Sci. U.S.A. 102, 9790 (2005).

[5] A. L. Hodgkin and A. F. Huxley, A Quantitative Description of Membrane Current and Its Application to Conduction and Excitation in Nerve, J. Physiol. 117, 500 (1952).

[6] R. W. Berg, M. T. Stauning, J. B. Sørensen, and H. Jahnsen, Comment on "Penetration of Action Potentials During Collision in the Median and Lateral Giant Axons of Invertebrates," Phys. Rev. X 7, 028001 (2017).

[7] See Supplemental Material at http://link.aps.org/ supplemental/10.1103/PhysRevX.7.028002 for further experiments on the collision of nerve pulses in the walking leg of lobster, and a discussion of pulse velocities. 